\title{
ON ENTIRE FUNCTIONS DEFINED BY A DIRICHLET SERIES: CORRECTION
}

\author{
Q. I. RAHMAN
}

1. As pointed out by Sunyer i Balaguer in the preceding paper the proofs of Theorem 1 and of the second part of Theorem 2 of our paper [1] are faulty. We observe that if we impose the additional hypothesis that $M_{S}(\sigma)=\max _{\left|t-t_{0}\right| \leq \pi a}|f(\sigma+i t)|(a>D)$, is a nonincreasing function for sufficiently small $\sigma$ then the proofs can be made to work. After correction Theorem 1 and the second part of Theorem 2 may be stated as follows.

Theorem A. If $M_{S}(\sigma)=\max _{\left|t-t_{0}\right| \leqslant \pi a}|f(\sigma+i t)|(a>D)$, is a nonincreasing function for sufficiently small $\sigma$ then the lower order $\lambda_{S}$ of $f(s)$ in each horizontal strip $S(\pi a)$, with $a>D$, is equal to the lower order $\lambda$ of $f(s)$.

TheOREM B. If $h=\infty, M_{S}(\sigma)=\max _{\left|t-t_{0}\right| \leq \pi a}|f(\sigma+i t)|(a>0)$ is $a$ nonincreasing function for sufficiently small $\sigma$ then the lower type $\tau_{S}$ of $f(s)$ in each horizontal strip $S(\pi a)$, with $a>0$, satisfies $\tau_{S} \geqq e^{-\pi \rho a} \tau$.

2. Proof of Theorem A. In the notations of $[1], \sigma_{j}^{*}=\sigma_{j}+k_{j}$, where $\left|k_{j}\right| \leqq \pi a$. By hypothesis $M_{S}(\sigma)=\max _{\left|t-t_{0}\right| \leqslant \pi a}|f(\sigma+i t)|(a>D)$ is nonincreasing for sufficiently small $\sigma$ and therefore for $\sigma_{j}<\sigma^{\prime}[1$, p. 215 , line 3 (correcting the obvious misprint)]

$$
\begin{aligned}
\log M_{S}\left(\sigma_{j}-\pi a\right) \geqq \log M_{S}\left(\sigma_{j}^{*}\right) & \geqq \log \mu\left(\sigma_{j}+P\right) \\
& -K>\log M\left(\sigma_{j}+P+\epsilon\right)-\log K_{1}-K .
\end{aligned}
$$

We can now conclude that $\lambda_{S} \geqq \lambda$. The fact that $\lambda_{S} \leqq \lambda$ completes the proof.

Proof of Theorem B. $M_{S}(\sigma)=\max _{\left|t-t_{0}\right| \leqslant \pi a}|f(\sigma+i t)|(a>0)$ is by assumption a nonincreasing function for sufficiently small $\sigma$ and therefore for $\sigma_{j}<\sigma^{\prime \prime}[1$, p. 215 , line 18]

$$
\begin{aligned}
\frac{\log M_{S}\left(\sigma_{j}-\pi a\right)}{e^{-\rho_{S}\left(\sigma_{j}-\pi a\right)}} \geqq \frac{\log M_{S}\left(\sigma_{j}^{*}\right)}{e^{-\rho_{S}\left(\sigma_{j}-\pi a\right)}} \\
\quad>e^{-\rho\left(\pi a+\epsilon^{\prime}+\epsilon\right)} \frac{\log M\left(\sigma_{j}+\epsilon^{\prime}+\epsilon\right)}{e^{-\rho\left(\sigma_{j}+\epsilon^{\prime}+\epsilon\right)}}-\frac{\log K_{1}+K}{e^{-\rho_{S}\left(\sigma_{j}-\pi a\right)}} .
\end{aligned}
$$

It follows that $\tau_{S} \geqq e^{-\pi \rho a} \tau$.

Received by the editors October 9, 1959. 
By the method of Sunyer i Balaguer [see Theorem A of the preceding paper] Theorem $\mathrm{B}$ can be improved and we can prove:

TheOREM C. Under the conditions of Theorem $\mathrm{B}, \tau_{S}=\tau$.

The following printing mistake in [1] may be noted:

In line 21 on page $215 e^{\rho\left(\sigma_{j}-\epsilon^{\prime}-\epsilon\right)}$ may be corrected to read $e^{\rho\left(k_{j}-\epsilon^{\prime}-\epsilon\right)}$.

\section{REFERENCE}

1. Q. I. Rahman, On entire functions defined by a Dirichlet series, Proc. Amer. Math. Soc. vol. 10 (1959) pp. 213-215.

NORTHWESTERN UNIVERSITY

\section{THE UNIVERSAL REPRESENTATION KERNEL OF A LIE GROUP}

\section{G. HOCHSCHILD}

Let $G$ be a connected real Lie group. The universal representation kernel, $K_{G}$, of $G$ is defined as the intersection of all kernels of continuous finite dimensional representations of $G$. Evidently, $K_{G}$ is a closed normal subgroup of $G$, and it is known from a theorem due to Goto (cf. [1, Theorem 7.1]) that $G / K_{G}$ has a faithful continuous finite dimensional representation. Thus $K_{G}$ is the smallest normal closed subgroup $P$ of $G$ such that $G / P$ is isomorphic with a real analytic subgroup of a full linear group. The known criteria for the existence of a faithful representation lead to a determination of $K_{G}$ which we wish to record here.

Suppose first that $G$ is semisimple. Let $\&$ denote the Lie algebra of $G$. Let $C$ stand for the field of the complex numbers, and denote by ${ }^{(5)}{ }^{c}$ the complexification of $(5)$, i.e., the semisimple Lie algebra over $C$ that is obtained by forming the tensor product, over the real field, of $\& 5$ with $C$. Denote by $S(\mathbb{S})$ and $S\left(\mathbb{S}^{C}\right)$ the simply connected Lie groups whose Lie algebras are $(S)$ and $B^{C}$, respectively. The injection $(B) \rightarrow B^{C}$ is the differential of a uniquely determined continuous homomorphism $\gamma$ of $S(\mathbb{B S})$ into $S\left(\mathbb{S}^{C}\right)$. The kernel $P$ of $\gamma$ is a discrete central subgroup of $S(\mathbb{S})$. Let $\phi$ denote the covering epimorphism of $S(\mathbb{S})$ onto $G$. We claim that $K_{G}=\phi(P)$, i.e., the universal representation kernel of the semisimple connected Lie group $G$ is the image, under the universal covering epimorphism, of the kernel of the canonical homomorphism $S(\mathbb{S}) \rightarrow S\left(\mathbb{S}^{C}\right)$.

Received by the editors September 18, 1959. 